\title{
Restarting the Information Society Based on Blockchain Technology
}

\author{
Marian STOICA, Bogdan GHILIC-MICU, Marinela MIRCEA \\ The Bucharest University of Economic Studies, Bucharest, Romania \\ marians@ase.ro,ghilic@ase.ro,mmircea@ase.ro
}

Social sciences literature claims that humanity is traversing a development stage generically called knowledge based informational society. It also anticipates another development horizon characterized by the explosion of artificial intelligence, corroborated with ever more spectacular development of information and communication technology. If we associate it with one of the most recent technologies dedicated mainly to information security - blockchain technology, we can certainly talk about a rebooting of informational society. Being itself a revolution, we will argue in this paper the level of influence blockchain has on business architectures, financial services, anti-plagiarism solutions, real estate transactions, economy, society, electoral processes and democracy as a whole. The ecosystem that supports all these transformations, developed and embedded in the global internet is IOT-Internet of Things.

Keywords: Blockchain, Knowledge Society, Internet-of-Things (IoT), Confidence Protocol, eVoting.

\section{1}

\section{Introduction}

The transformations inflicted upon the current human society are no different in scope from the ones in the past. What makes them different is the propagation speed over big distances and fast adoption in almost every country. The new globalization economic trend has accelerated the changes, companies seeing the trans-national and transfrontier idea as an opportunity to develop through new markets, cheaper or better qualified workforce, cheaper and easier accessible raw materials. Business prospects have stimulated companies to invest more in data gathering and transmission infrastructure, which led to a more intense research in the field of informatics and telecommunications, which in turn led to an even more increased impact of these technologies upon human society.

Currently, the challenges of knowledge society, global economic crisis, frequent changes on business environment and implicitly on workforce market require an internet trust protocol that meets the new requirements and changes as well as the future development directions of a technological era. This trust protocol for internet identified in the blockchain technology must be used to build an infrastructure that allows the individual to acquire the following professional competencies (without being limited to them):

$\checkmark$ Use modern theories, principles, methods and instruments for analysis of knowledge, objectives, opportunities, benefits, risks, quality, efficiency, innovation in the context of knowledge society;

$\checkmark$ Apply modern concepts, principles and paradigms of the knowledge society in various fields in order to ensure sustainability and innovation;

$\checkmark$ Use ICT to model, design, implement and manage activities and new organizational paradigms specific to the knowledge society;

$\checkmark$ Develop policies that ensure the implementation of newest methods, techniques, paradigms and technologies in knowledge society's economy;

$\checkmark$ Scientific research and design of organizational architectures for informational optimization of the context of knowledge society;

$\checkmark$ Update and innovate methods and techniques for scientific research in functional infrastructure of virtually organized activities in digital economy.

As argument, we must note that the last two decades are characterized by a series of processes and phenomena that indicate 
humanity is in a transition phase of its evolution from industrial society to another type of society. Industrial society emerged with the industrial revolution that meant introduction of machines in economy, social division of work and creation of assembly lines. These elements have led to a huge leap forward for society, with implications not only in economy but also in social, cultural and political life. Resources that lie at the base of this society are energy and raw materials. Economic activity is the ensemble of human actions and decisions regarding rational use of resources to create goods and services, according to the needs and economic interests. Informational and knowledge society is based on another resource, which takes the main stage: information. This doesn't mean that industrial society does not use information or knowledge, but that technological explosion of the last years has turned information into a strategic resource, a new production factor. The speed of knowledge renewal and the exponentially increasing volume have made knowledge an important component of modern economy and a basic organizational principle of the new society. Knowledge has become the most important production factor in modern economy. It constitutes the basis of power exercise, generates increased productivity and ensures business competitiveness. Since and economy can be defined by the type of activity involving most of its worker, we can currently speak of an informational and knowledge economy just as we spoke about industrial economy (when production and processing were dominant activities) or agricultural economy (when most workers were involved in agriculture).

\section{Context of Blockchain Technology Emergence}

The apex of informational technology was not even reached yet and people are already asking: what is next? Studies establish a reference point for informational society the next 20 or 30 years. The question is what new technologies will emerge and follows after this interval. A starting point could be the so called calm technologies and another is the invisible technologies, mind power technologies and bio (mechanical, informational, magnetic etc.) or eco technologies [1]. Futurologists have to find credible answers for all these questions. Analyzing the society evolution through the classical data-information-knowledge trident, we can talk about knowledge society and intelligent society. The current state of humanity development may be defined as a new type of society, in a continuous change, developed on the production of informational values, unlike previous societies which were driven by material production. From this we may foresee that the next wave will start around year 2035-2040 and it may be called intelligence and knowledge stage. It will shift the focus on exploiting information to achieve the intelligence level desired by an entity. It will be the time when the capabilities of the human brain will be matched by technology and the concept of bio-techno-system will be generalized - hybrids between biological and technological systems.

This is the current cognitive and technological context, where technology no longer creates prosperity and it doesn't destroy the private life either. Still, in this digital age, technology can be seen in every aspect of human life, at the core of everything good or bad. It is the technology that provides individuals with the possibility to reciprocally value and infringe the other's rights in new and deeper ways [2]. Three years ago we were writing about redefining telework through the cloud computing paradigm [3], highlighting the very important role of trust in the employeremployee relation (Romanian law for regulating telework activities was finally adopted in 2018 - law 18/2018 and it does not mention at all the aspect of trust, teleworking being based only on the accord between the parties). In the context of digital age, security breaches in informational systems and ICT vulnerabilities in current systems (electronic mail, social networks, professional networks and so on) unavoidably affect the trust. 


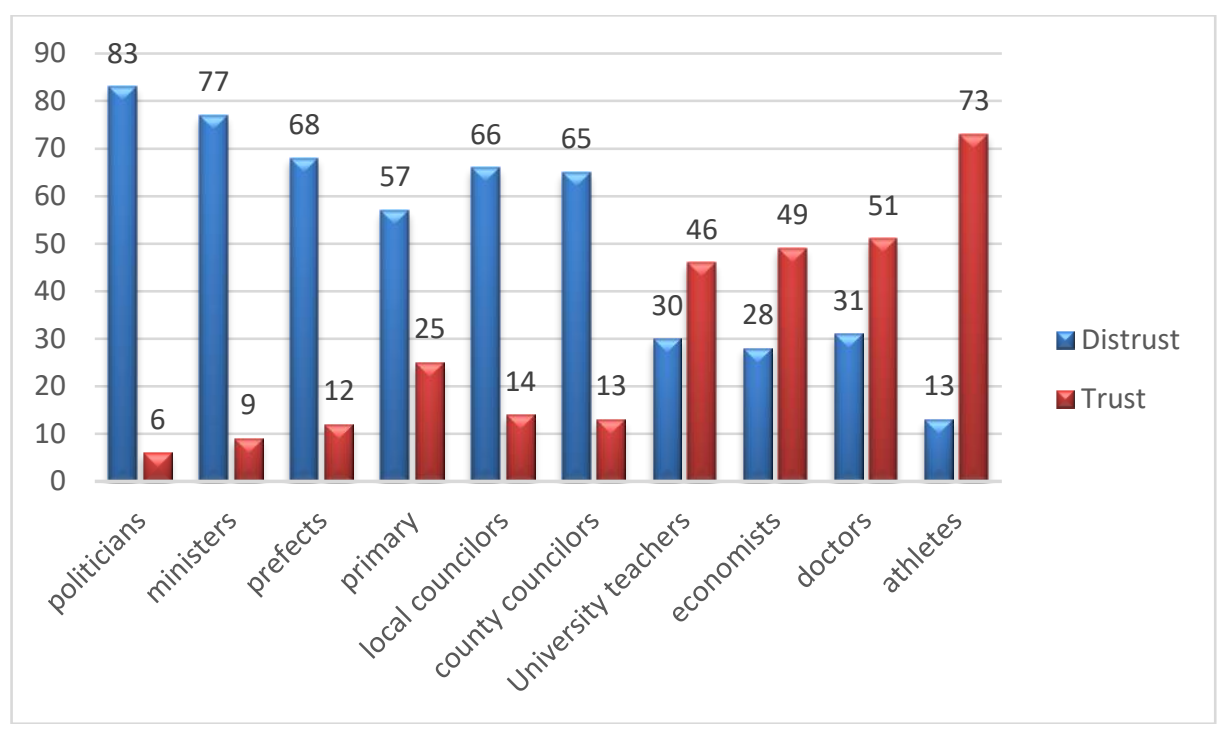

Fig. 1. Trust poles for public entities in Romania

From a business perspective, in the digital age, gaining trust means the partner acts according to the four principles of integrity: honesty, consideration, responsibility and transparency. For example, the trust barometer created by the public relation company Edelman in 2018 shows a record decrease of US population trust in public institutions, down to the levels reached during the 2008 recession. Even the ICT industry, once impregnable and with the highest trust level over many years, is now on a downwards trend and seen significant decreases in most countries [4]. The same can be seen in Romania, according to studies published by public polling institutions - for example by Romanian Institute for Evaluation and Strategy (IRES) [5]. On the top of lack of trust sit the public institutions (parliament, government, local government offices, city halls etc.). Highest trust is given to university professors, economists, doctors etc. (figure 1). On the other hand, the same source (Edelman) shows that in January 2019 the trust poles have changed drastically in the last year. People have changed the trust regarding the work relations, especially towards their employers. Globally $75 \%$ of people trust that "my employer" does the right thing, significantly higher than for NGOs (57\%), business environment $(56 \%)$ and mass-media $(47 \%)[6]$.
In the pre-blockchain context, transaction trust came from the integrity of involved persons, intermediaries or other organizations. When knowledge about partners and their integrity is lacking, we rely on intermediaries that vouch for the unknown parties, keeping records of transactions that feed the online commerce. These records end up in the hands of giant digital corporations like banks, governments, Google, Facebook Amazon, Apple, Uber, Visa etc.

Al this highlights an acute lack of trust in general and strongly supports the need for trust. In the emergent blockchain world trust derives from the network, from the many blocks that form the chain. In this case trust is related to the integrity and protection of information, not trust in the business endeavors. In other words, anticipating the evolution of production neo-factors from the perspective of modern management, we may argue that those businesses doing their transactions, in part or completely, through blockchain technology will be gain a strategic advantage regarding trust. Trust will take a seat next to information, technology, brand or organizational culture as a vital emerging production factor in a society of knowledge. Blockchain is a protocol that can help internet (re)gain trust. 
3. What is Blockchain and How Does It Work?

Blockchain technology was invented by a person (or group pf persons) known as Satoshi Nakamoto and published in 2008. The main advantage of this technology is that allows distributing information but not copying it. First field to take advantage of this was cryptocurrency (bitcoin in 2009), but its applicability is more and more desired in other fields. The general understanding is that blockchain is an incorruptible collection of transactions that can be programed not only to record economic exchanges but any virtual values.

In what concerns the inner working of blockchain, the answer might seem complicated, but is can be easier to understand by analogy to a spreadsheet. The sheet may be duplicated millions of times in a network of computers, called nodes, which are programed to update the sheet regularly. In essence, this is the basis of blockchain. As a data structure, blockchain is a linked list, where the links are represented by a summary of block informational content (hash). Thus, each block contains a link to a previous block (a hash of the previous block), a digital stamp with date and time of operations (timestamp) and transaction data (Figure 2).

Another way to see blockchain is as a database distributed in each node. Because the database is stored on many computers, the records are public and easy to verify. There is no centralized version of these records, so they cannot be altered by a hacker. Another advantage comes from the fact that there is no unique entry point, since the database is distributed on many nodes.

The biggest accounting companies, Ernt\&Young, KPMG, Delloite and Pricewaterhouse Coopers are testing blockchain technology under various forms. Ernt\&Young has installed an ATM in their Swiss branch and accepts payments in cryptocurrencies for their consulting services. KPMG, Delloite and Pricewaterhouse Coopers are testing this technology in a private environment.

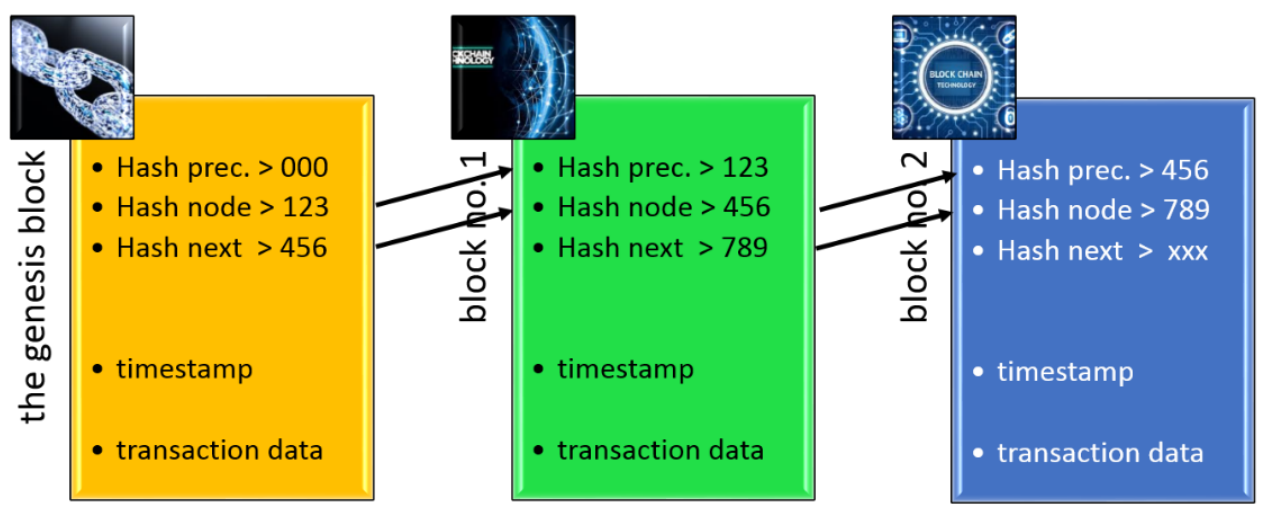

Fig. 2. Blockchain mechanism

Using blockchain technology, any transaction attribute or information about agents and goods involved is stored in a distributed registry and may be accessed in real-time. The trust in the intermediary is replaced by trust in the basic code and consent rules. These rules define the way a distributed network may reach an agreement, at regular intervals, concerning the status of the shared data it must maintain in order to work in a digital market. Blockchain technology allows a network of economic agents to agree, on regular intervals (10 minutes - the time to create a block) about the real status of shared data. The flexibility of the information represented by these shared data creates a technology with general purpose.

While it is often compared with communication protocols which concentrate on the way information is packaged and directed though the internet (see TCP/IP protocol), blockchain technology is 
fundamentally different, because it allows safe transfer and execution of ownership rights. This allows the creation of new types of platforms for trading and providing digital assets is not based on an intermediary. On these platforms, the trust in the platform operator is replaced by the trust in incentives, code and consensus that constitute is foundation. As a result, the intermediary market powers, confidentiality risk and censorship risk are low.

\section{Solutions and Possible Implications of Blockchain}

We must say that blockchain technology is not exclusively owned by the financial industry (figure 3). There are platforms destined to online writers, with the purpose of guaranteeing the authenticity of texts and fight plagiarism. One such example is the MAVEN project, which is a coalition of experts functioning on an online platform for publishing, publicity and digital distribution, unified under a single media brand [8]. Tens of prizewinning journalists, best writers, sports journalists, top analysts, big causes and foundations bring their organizations in this elite coalition. There are many such antiplagiarism solutions based on blockchain and China is the country that implements them on large scale. These solutions are even embedded in juridical system. According to [9], a court of law recently established in the city of Hangzhou, east China, is set to make use of blockchain technology in the fight against plagiarism for online writers. The use of this technology will save time and reduce the general costs for justice.

Another pioneering element was launched in November 2018 in Foshan, Guangdong province, south China: the first community correction service system based on blockchain to manage the criminals in Chancheng district in real time. The system allows the local police, prosecutors and magistrates to share data in real time and coordinate actions for a better surveillance and services regarding criminals freed on parole or bail.

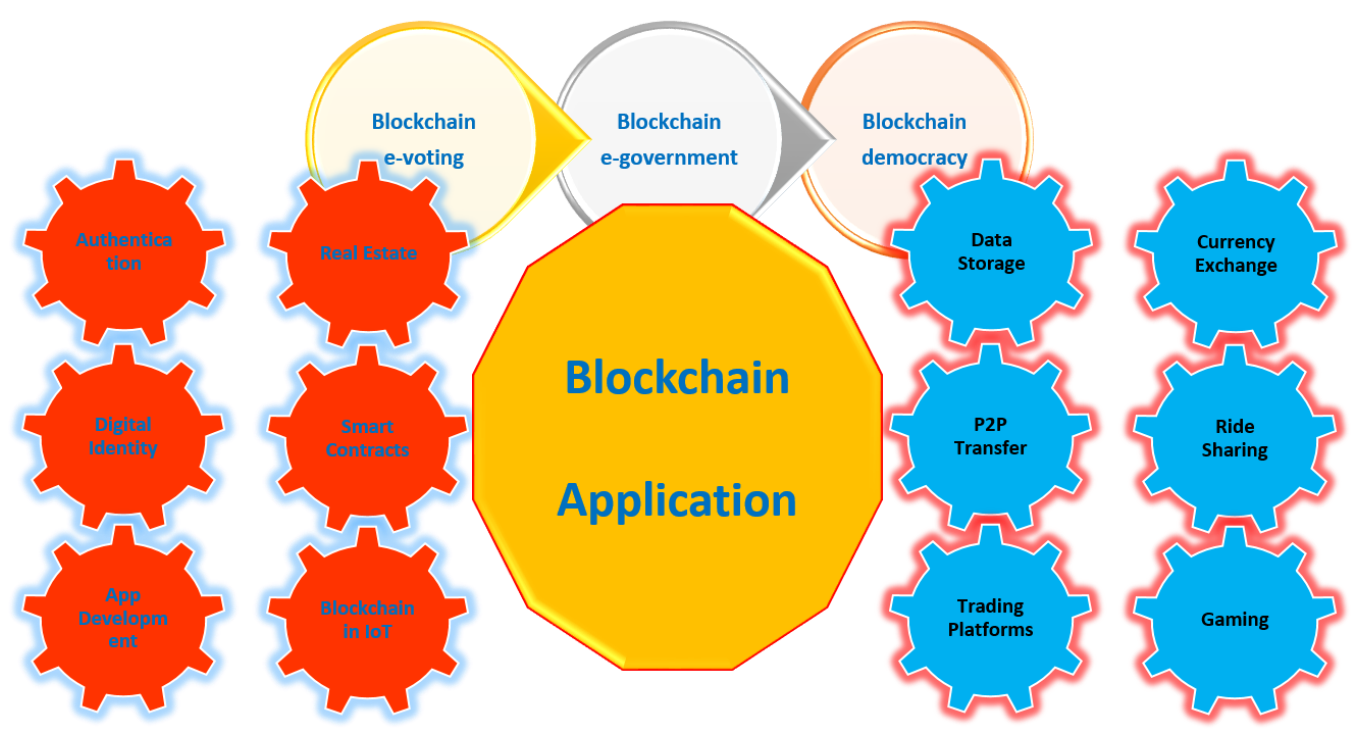

Fig. 3. Blockchain based applications

Unfortunately, plagiarism exists in every sphere of innovation. In 2018 thousands of blockchain companies emerged and plagiarism scandals became a daily issue. Even success projects are exposed to such accusations, according to [10]. Another performant anti-plagiarism solution is CERTO [11] - an application that uses blockchain technology to verify the existence of any document with a given date in order to prove the authenticity of a product, brand, text and digital literature, video clips or still images, art, illustrations, cartoons, software and so on. CERTO can provide an authenticity certificate by recording the document in the block repository, against plagiarism, fakes 
and author rights infringements, incorrect use and illegal distribution.

From the current public blockchain platforms dedicated to open source applications worthy mentions are NXT Blockchain Platform (available since 2013) [12] and Ardor Blockchain Platform (available since 2018) which provides features under the Blockchain-as-a-Service policy [13].

The implications of blockchain are and can go much deeper: from redesigning business processes and enterprise architectures to electronic government, e-voting, digital democracy or liquid democracy. Tapscott speaks of initializing the future (adaptation of the term bootstrapping - the process of initializing an automated sequence or, in this case, of a blockchain) and the seven principles of blockchain economy: network integrity, distributed power, value as incentive, security, confidentiality, rights conservation and inclusion [2].

No matter which side we take regarding blockchain we must be aware of the size of its ecosystem. The main actors of this ecosystem are governments, regulating authorities, NGOs, banks and financial services, risk capital investors, academic environment, developers and users.

\section{Blockchain Technology and Real Estate Transactions}

Many fields of activity and many industries have recently discovered the advantages of blockchain technology. One such industry, dealing with the largest class of assets in the world is the real estate industry [14]. According to the research division of British company Savills (one of the largest real estate companies in the world, with over 160 years of experience [15]), in order to realize the correct dimension of real estate context we may compare the total value of the gold ever mined (approximately 6 trillion USD) which is shadowed by the total value of real estate developed world wide (which is at least 36 times larger). We cannot ascertain how objective is Savills evaluation but the size of the global real estate industry is easy to gauge. In other words, the real estate is the class of assets that is most likely to be affected by global monetary conditions and investment activity. In turn, it has the power to make the largest impact on national and international economies. In simpler terms, the fortune has and will continue to have a huge role in global economy. In spite of all these realities, just like other traditional industries, the real estate industry is severely impacted by at least six factors:

1. Restrictive access to market

2. Major lack of transparency

3. Very high taxation

4. Lack of liquidity (understood as transforming the real estate into money)

5. Commission levels

6. Transaction speed

Considering all these factors, we can easily understand why the real estate industry is vulnerable and prone to perturbations. This is where blockchain technology comes in.

As we have seen, in simplest terms, a blockchain is a series of immutable data records, administered by a group of computers that does not own any entity. Each of these data blocks is secured and interlinked using cryptographic principles (effectively forming a chain). From the real estate industry point of view, the blockchain technology brings three fundamental features (figure 4):

1. Decentralization - data stored in a blockchain are not owned by a single entity;

2. Transparency - data stored in a blockchain may be viewed by all members of the network (each piece of information can be tracked to its origin);

3. Immutability - data inside a blockchain cannot be changed because of the cryptographic components (mainly security hash functions). 


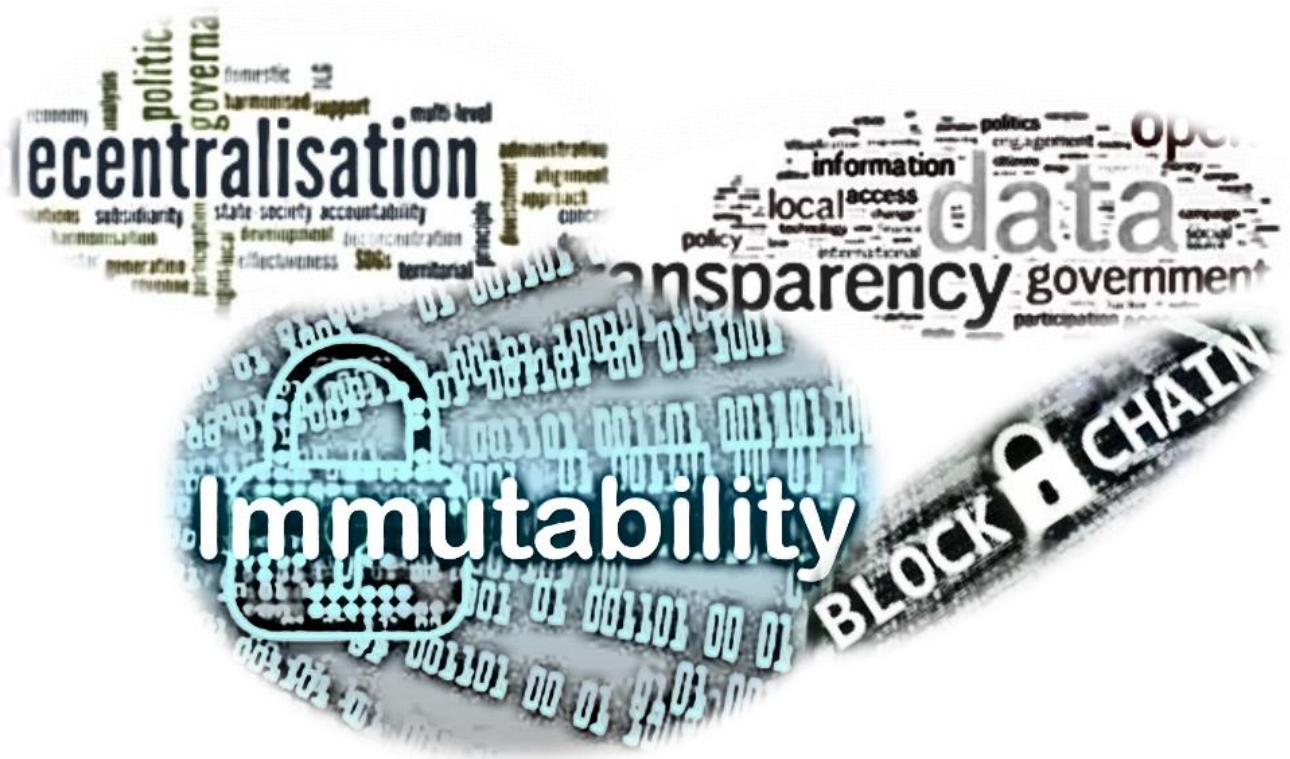

Fig. 4. Basic features of blockchain technology

Considering these features of the paradigm, blockchain brings several advantages in the real estate ecosystem, including intelligent contracts and tokening. Intelligent contracts are automated contracts. They execute automatically with specific instructions embedded in their code, triggered when certain conditions are fulfilled. Intelligent contracts are made of a series of instructions written in a specific programming language working in the logic of If-This-Then-That. When the first set of instructions is completed, the next function is executed, then the next one and so on until the end of the contract. The most obvious benefit of intelligent contracts is the elimination of all intermediaries. With all brokers, banks, lawyers bypassed a lot of fees and commission money will be saved. Thus, intelligent contracts will considerably accelerate real estate transactions.

According to Wikipedia, "tokening, when applied to data security means the process of replacing a sensitive data item with a nonsensitive equivalent, called a token, which has no inherent meaning or extrinsical or exploitable value. The token is a reference (an identifier) that maps back to the sensitive data through a tokening system". In simple terms, a token is a digital representation of an asset, value or function from the real world. One of the most interesting cases of using blockchain technology is to symbolize real world assets.
This tokening not only increases the liquidity of traditionally non-liquid assets (properties), but also makes possible the transaction of such assets without a third party (in other words, instead of buying a property, we buy tokens). The token in blockchain space fall under three categories:

1. Payment currency - cryptocurrency which is already largely known: Bitcoin, Litecoin, Ethereum, Bitcoin Cash, Stellar, XRP etc. (may be used as currency inside or outside of their respective platforms)

2. Utility tokens - which fulfill a certain utility of function in their respective platforms: may give the owners the right to use the network and/or profit of the network by voting inside the ecosystem

3. Security tokens - which derive their value from an external tradeable asset: they are securities and are regulated (this is the category used to symbolize real estate assets) One of the most interesting results of tokening is fractioned property. This is very interesting for expensive assets, like real estate. Instead of one person owning a property, several persons may buy tokens for the property and co-own it. This is in fact a sharing the rights over a real estate asset.

Blockchain technology will allow the democratization of real estate. This will open the gates for potential investors world-wide to try their luck in real estate investments. 


\section{Conclusions}

Blockchain has become a revolutionary solution for peer-to-peer (P2P) computing a few years ago. Some recognize blockchain as a technology born out of bitcoin, the first major cryptocurrency. Bitcoin was a challenge for the banking industry, but blockchain will change the world in the next 10-15 years more than cloud, robotics or other fast growing technologies. Blockchain analysis has managed to create a new world without intermediaries. Companies can exploit the blockchain technology to build wide computation systems, distributed and open to everybody. Trust is embedded in the blockchain network, each transaction being recorded anonymously in the distributed registry or database. The records block changing the payment, music file, medical record or any other file and highlight them in chronological order for ever. No user can secretly modify them because each transaction block must link to the previous block to be valid. This approach has the potential to reorient the entire data and cloud computing industry on short term. Companies and individual consumers may soon store their data in distributed cloud network, based on extremely safe blockchain technology. Cloud computing already brought lower costs, but P2P networks consisting of thousands of personal computers and servers all over the globe bring open cloud computing to an accessible price level for everybody. Thus, we might easily imagine the way computer programs will work on this new infrastructure, which is currently used to validate transactions. We cannot literally accept the computerized analogy in cloud, because block infrastructure cannot completely replace cloud computing, although it divides and democratizes the cloud.

In conclusion, we may admit and agree the idea that we live the beginning of blockchain, but perspectives are interesting. Industry expert Don Tapscott considers this is a real paradigm shift [7]. His point of view is that blockchain may revolutionize world economy by leveling the playing field, no matter the economic status of each participant and it may create new opportunities to generate added value.

This plus-value may come from the real estate industry itself through adoption of blockchain technology. At first sight it may seems fantastic, but it is not impossible. For example, according to the World Economic Forum of January 21-24, 2015 at DavosKlosters, Switzerland, in the following 10 years (so until 2025) 10\% of world GDP will be stored in cryptographic assets. Thus, the value of token assets will be around 10 trillion USD. Also, the World Economic Forum indicated that if blockchain becomes a mass technology, it is likely that the traditional role of governments and institutions will be reimagined: "economy and monetary management will be reviewed by new systems anchored in digital currencies and blockchain, which will make traditional mechanisms of pricing and exchange less relevant".

\section{References}

[1] M. Stoica, M. Mircea, B. Ghilic-Micu, C. Uscatu, From a Smart Education Environment to an Eco-School through Fog \& Cloud Computing in IoT Context, Informatica Economică Journal, Vol. 22, no. 4/2018, pp. 5-14, ISSN 1453-1305

[2] D. Tapscott, A. Tapscott, Blockchain Revolution, Ed. Act and Politon, Bucharest 2017, ISBN 978-606-913-2586

[3] B. Ghilic-Micu, M. Stoica, A Redefinition of Telework Through Cloud Computing Telework 2.0, Bulletin of Taras Shevchenko National University of Kyiv. Economics, Ucraine, Vol. 46, no. 188/2016, pp. 16-21, ISSN 1728-3817

[4] https://www.iqads.ro, Barometrul de Încredere Edelman 2018, https://www.iqads.ro/articol/41399/baro metrul-de-incredere-edelman-2018-aratao-scadere-record-a-increderii, [September 10, 2018]

[5] Barometrul încrederii românilor, IRES 2014-2015, http://www.ires.com.ro, Available: 
http://www.ires.com.ro/uploads/articole/b arometrul-increderii-romanilor.pdf, [May $12,2018]$

[6] $2019 \quad$ EDELMAN TRUST BAROMETER, January 20, 2019, Available: https://www.edelman.com/trustbarometer, [March 10, 2019]

[7] Tapscott Group CEO Don Tapscott, How blockchains could change the world, Available: https://www.mckinsey.com/industries/hig h-tech/our-insights/how-blockchainscould-change-the-world, [September 11, 2018]

[8] https://mavencorp.io/press/mavenpartners-with-blockchain-based-po-et-toempower-publishersuLPMlewMKkqaKKsqsghxOQ/

[9] Chinese Internet Court: Blockchain against online plagiarism, December 19,
2018, https://cryptoheroes.ch/chineseinternet-court-blockchain-against-onlineplagiarism/

[10] The Loudest Blockchain Plagiarism Scandals, Available: https://www.tokendesk.io/the-loudestblockchain-plagiarism-scandals/ [January 4, 2019]

[11] CERTO Legal, http://www.certo.legal/en/index.html, [February 9, 2019]

[12] Ardor Cryptocurrency and Blockchain Technology, https://ardorplatform.org/

[13] Nxt - The Blockchain Application Platform, https://nxtplatform.org/

[14] Rajarshi Mitra, Blockchain Real Estate How Will Blockchain Change Real Estate?, Available: https://blockgeeks.com/guides/blockchain -real-estate/ [May 10, 2019]

[15] The experts in local and international property, https://www.savills.com/

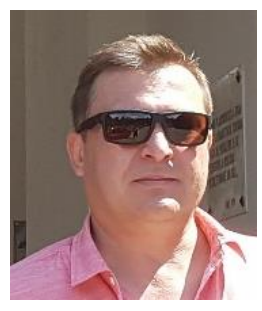

Marian STOICA received his degree on Informatics in Economy from the Bucharest University of Economic Studies in 1997 and his doctoral degree in economics in 2002. Since 1998 he is teaching in Bucharest University of Economic Studies, at Informatics and Cybernetics Economy Department. His research activity, started in 1996 and includes many themes, focused on management information systems, computer programming and information society. The main domains of research activity are Information Society, EActivities, Tele-Working, and Computer Science. The finality of research activity still today is represented by over 80 articles published, 25 books and over 40 scientific papers presented at national and international conferences. Since 1998, he is member of the research teams in over 30 research contracts with Romanian National Education Ministry and project manager in 5 national research projects.

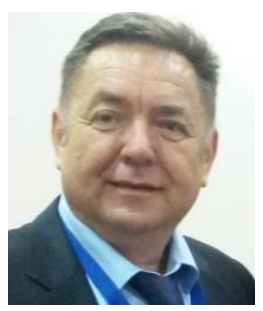

Bogdan GHILIC-MICU received his degree on Informatics in Economy from the Academy of Economic Studies Bucharest in 1984 and his doctoral degree in economics in 1996. Between 1984 and 1990 he worked in Computer Technology Institute from Bucharest as a researcher. Since 1990 he teaches in Academy of Economic Studies from Bucharest, at Informatics in Economy Department. His research activity, started in 1984 includes many themes, like computers programming, software integration and hardware testing. The main domain of his last research activity is the new economy - digital economy in information and knowledge society. Since 1998 he managed over 25 research projects like System methodology of distance learning and permanent education, The change and modernize of the economy and society in Romania, E-Romania - an information society for all, Social and environmental impact of new forms of work and activities in information society. 


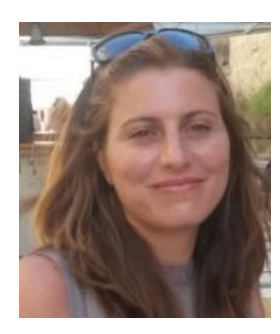

Marinela MIRCEA received her degree on Informatics in Economy from the Academy of Economic Studies, Bucharest in 2003 and his doctoral degree in economics in 2009. Since 2003 she is teaching in Academy of Economic Studies from Bucharest, at Informatics and Cybernetics Economy Department. Her work focuses on the programming, information system, business management and Business Intelligence. She published over 40 articles in journals and magazines in computer science, informatics and business management fields, over 30 papers presented at national and international conferences, symposiums and workshops, she was member over 15 research projects and project manager in 2 national research projects. She is the author and co-author of 12 books. In February 2009, she finished the doctoral stage, and her $\mathrm{PhD}$ thesis has the title Business management in digital economy. 